\title{
PROCESSO DE TRANSFORMAÇÃO ÁGIL EM UMA EMPRESA BRASILEIRA DE TELECOM
}

\author{
AGILE TRANSFORMATION PROCESS IN A BRAZILIAN TELECOM COMPANY
}

Marcelo Luiz do Amaral Gonçalves

Mestre em Gestão de Projetos

Universidade Nove de Julho - Uninove

São Paulo, SP - Brasil

marluago@gmail.com

Ricardo Antônio Câmara da Silva

Mestre em Gestão de Projetos

Universidade Nove de Julho - Uninove

São Paulo, SP - Brasil

rcamara62@gmail.com

Eduardo André Cândido Silva

Especialista em Gestão e Governança de TI

Universidade Nove de Julho - Uninove

São Paulo, SP - Brasil

eduardoandre@hotmail.com

\section{Resumo}

O objetivo deste artigo é identificar os processos de gerenciamento aplicados nos projetos, os obstáculos e os facilitadores da adoção dos métodos ágeis no contexto de transformação digital em uma empresa brasileira de Telecom. A empresa decidiu mudar seu modelo de gerenciamento de projetos para se adequar às necessidades de mercado, adotando um framework de gerenciamento e ferramentas ágeis. A unidade analisada foi o departamento de infraestrutura de TI. Os dados foram coletados por meio de pesquisa documental, entrevistas e observação participante. Os resultados evidenciaram o estágio de transição entre o modelo tradicional e o modelo ágil, caracterizando-se como uma gestão híbrida de projetos. Os processos da fase de execução, com entregas particionadas, adequam-se às práticas e métricas ágeis. Nas demais fases ainda se aplica o modelo tradicional, vinculado a processos obrigatórios e a exigências de documentação. Foram identificados fatores facilitadores e barreiras ao processo de transformação ágil. Verificou-se que o framework híbrido de gestão de projetos adotado é aderente ao Modelo-V, descrito na literatura. Embora limitado a uma área específica de uma empresa. O estudo contribui com o conhecimento sobre a convivência de abordagens gerenciais na transformação ágil e como fonte de lições aprendidas para outras empresas.

Palavras-chave: Transformação ágil. Gerenciamento ágil de projetos. Gerenciamento tradicional de projetos. Gerenciamento híbrido de projetos. Frameworks híbridos. 


\begin{abstract}
The objective of this article is to identify the processes of managing the knowledge in projects, the objectives, and the facilitators of the adoption of agile methods in the context of digital transformation in a Brazilian Telecom company. The company decided to change its project management model to suit market needs, adopting a management framework and agile tools. The analysis unit was the IT infrastructure department. The data were collected through documentary research, interviews, and participant observation. The results showed the transition stage between the traditional model and the agile model, characterized as a hybrid project management. The processes of the execution phase, with partitioned deliveries, are adapted to agile practices and metrics. In the other phases, the traditional model still applies, linked to mandatory processes and documentation requirements. Facilitating factors and barriers to the agile transformation process were identified. It was found that the hybrid project management framework adopted is adherent to the V-Model, described in the literature. Although limited to a specific area of a company, the study contributes with knowledge about the coexistence of managerial approaches in agile transformation, and as a source of lessons learned for other companies.
\end{abstract}

Keywords: Agile transformation. Agile project management. Traditional project management. Hybrid project management. Hybrid frameworks.

\title{
Cite como - American Psychological Association (APA)
}

Gonçalves, M. L. do A., Silva, R. A. C. da, Silva, E. A. C., \& Penha, R. (2021, Ed. Esp. jan./abr.). Processo de transformação ágil em uma empresa brasileira de Telecom. Revista de Gestao e Projetos (GeP), 12(1), 70-94. https://doi.org/10.5585/gep.v12i1.17801.

\section{Introdução}

Os projetos de Tecnologia da Informação (TI) possuem uma gama de aplicações, incluindo desenvolvimento de software, sistemas de informação e implantação de infraestrutura. Executados em um contexto de desenvolvimento tecnológico, o ambiente de negócios e os requisitos estabelecidos por empresas e usuários desse segmento mudam com frequência e de forma inesperada (Babenko et al. 2019; Rahmanian 2014). Tais mudanças contribuem para instabilidade em processos importantes dos projetos, como o gerenciamento do prazo e do custo, com impacto direto no cronograma e no orçamento (Rahmanian, 2014). As abordagens tradicionais de gerenciamento não se mostraram suficientes para responder às dinâmicas mudanças nos mercados, tecnologia ou ambientes de negócios (Shenhar, 2012). Nesse contexto, o gerenciamento ágil é considerado como uma prática mais adequada para tratar a gestão de mudança e seus impactos nos projetos (Aguanno, 2005; Conforto et al., 2014).

O número de organizações que está utilizando os modelos ágeis de gerenciamento em seus projetos é crescente por meio de um processo que está sendo conhecido como "transformação ágil", o qual vai além do gerenciamento e promove a mentalidade ágil a nível organizacional, impactando processos, cultura e modelo de negócios (Paterek, 2018). 
No âmbito dos projetos de TI, por exemplo, as abordagens de gerenciamento ágil vêm sendo usadas de alguma forma por $95 \%$ das equipes de software em diversos ambientes (Sweetman \& Conboy, 2018). Autores como Boehm (2002), entretanto, defendem que nem todos os projetos em uma organização podem ser totalmente ágeis, afirmando que cada abordagem tem elementos de melhor desempenho e, portanto, é preferível uma abordagem combinada.

Nesse contexto, a adoção da agilidade em larga escala exige a integração de elementos ágeis e não ágeis para a construção de uma metodologia adaptativa híbrida. Surge, assim, o desafio de determinar quais os elementos relevantes desse processo de transformação e como adaptar para eles as práticas ágeis (Dyba \& Dingsoyr 2009; Gill et al., 2018; Highsmith 2009). Pesquisadores como Vinekar et al. (2006) e Cram e Marabelli (2018) ressaltam a importância de se examinar esse dinâmico ambiente durante a transição para os métodos ágeis, quando as organizações são desafiadas a equilibrar áreas aparentemente conflitantes sem sacrificar os benefícios associados à metodologia tradicional, mesmo cientes do risco de precisar fazer concessões, de forma tal que nenhuma das abordagens tenha desempenho eficaz.

A literatura recente ainda não se concentrou nessas "transições" centradas no conhecimento, que envolvem uma mudança das metodologias tradicionais para as ágeis nos processos ainda em andamento. A abordagem dessa lacuna teórica, porém, é extremamente relevante para os profissionais, particularmente aqueles com pouca experiência nas práticas ágeis (Kusters et al., 2017; Cram \& Marabelli, 2018).

Considerando o acima exposto, este artigo pretende responder à questão de pesquisa: "Como ocorre a transição de uma abordagem tradicional para uma abordagem ágil em uma empresa de Telecom durante o processo de transformação ágil?". Dessa forma, o objetivo deste artigo é identificar os processos de gerenciamento aplicados nos projetos, os obstáculos e os facilitadores da adoção dos métodos ágeis no contexto de transformação digital em uma empresa brasileira de Telecom. O propósito final da empresa foi realizar uma transformação ágil, incutindo a cultura de agilidade para melhorar a entrega de valor aos clientes e manter-se competitiva no mercado.

O artigo está estruturado sob a abordagem qualitativa descritiva e utilizou como estratégia de pesquisa o estudo de caso único para investigar o objeto e responder à questão de pesquisa. Para observação, foi escolhido um recorte nos processos de gerenciamento dos projetos da empresa na área de Infraestrutura de TI, para identificação das atividades, processos ou fases dos projetos que estão em formato ágil ou tradicional.

\section{Referencial teórico}

A seguir é apresentada uma breve revisão da literatura existente sobre: métodos 
tradicionais e ágeis de gerenciamento de projetos, transformação ágil e frameworks híbridos.

\subsection{Métodos tradicionais}

Os princípios norteadores das primeiras técnicas de gerenciamento de projetos delinearam o modelo denominado plan-driven ou orientado ao planejamento. Nesse contexto, assume-se que os projetos são previsíveis e os gestores devem prever as atividades, estimar necessidades e restrições, elaborando um planejamento detalhado inicial, que norteia todas as atividades do projeto, sem grandes mudanças (Boehm \& Turner, 2003; DeCarlo, 2010; Shenhar \& Dvir, 2010; Wysocki, 2019). Esse tipo de abordagem preza pela eficiência operacional, administração e controle das restrições do escopo, do prazo, do custo e da qualidade, com padrões e documentação abrangente (Boehm, 2002; Shenhar, 2012). Essas práticas tinham como pressuposto a possibilidade de gerir projetos de qualquer natureza, por meio de um conjunto de ações, técnicas e ferramentas, padronizadas nos chamados "guias de conhecimento", como PMBoK, ISO e IPMA. Foram largamente disseminadas e utilizadas, especialmente em gerenciamento de software, sendo hoje classificadas como "Modelo Tradicional" (Silva, Bianchi, \& Amaral, 2019). Seguem um processo sequencial para cada fase do ciclo de vida do produto.

Os projetos orientados ao planejamento, executados para desenvolvimento de produtos, apresentaram falhas relacionadas às pressões relacionadas ao tempo para colocar o produto no mercado (Leffingwell, 2011). Novos modelos, mais iterativos, foram então desenvolvidos. Denominados “incrementais", neles o progresso acontece por meio de sucessivos refinamentos, melhorados a cada iteração (Sommerville, 2011; Pressman \& Maxim, 2016). Apesar desses modelos ainda seguirem etapas, passou a existir uma maior preocupação com o entendimento dos requisitos, os processos de feedback e as descobertas ao longo de todo o projeto. Com o tempo, esses modelos evoluíram para os modelos conhecidos hoje como ágeis (Bianchi, 2017).

\subsection{Métodos Ágeis}

O termo Gerenciamento Ágil de Projetos (GAP) surgiu no início dos anos 2000 com origem na comunidade internacional de desenvolvimento de sistemas de informação na busca por soluções que levaram ao desenvolvimento de abordagens alternativas (Beck et al.,2001; Amaral et al., 2011). Tratase de um processo altamente iterativo e incremental, no qual os membros do time e os demais interessados no projeto trabalham juntos, para entender o domínio do problema, identificar o que precisa ser construído e priorizar as funcionalidades (Babenko et al., 2019). Algumas das principais características dos métodos ágeis estão no incentivo ao envolvimento do cliente durante o projeto, no 
alto grau de novidade, num escopo que não é de todo conhecido e que aceita mudanças durante os ciclos, no alto nível de incerteza, nos objetivos não muito claros e nas mudanças constantes durante o desenvolvimento (Amaral et al., 2011; Chin, 2004; Highsmith, 2009; Augustine, 2005).

Portanto, nos últimos anos, diversas organizações de projetos, principalmente as de software, começaram a explorar modernas abordagens de gestão a fim de desenvolver a agilidade organizacional e melhorar sua posição no mercado dinâmico e imprevisível (Gurd \& Ifandoudas, 2014; Cegarra-Navarro et al., 2016; Paterek, 2018; Ravichandran, 2018). Assim, começaram a adotar metodologias ágeis de gerenciamento de seus projetos, a fim de lidar melhor com o planejamento e a execução da complexidade, num processo conhecido como "transformação ágil" (Paterek, 2018).

\subsection{Transformação Ágil}

O conceito de "transformação ágil" é conhecido por diferentes termos como "transição ágil”, "implantação ágil”, "adoção ágil” ou "mudança organizacional ágil". Vai além de mudanças de metodologias e processos do gerenciamento de projetos, incluindo mudanças organizacionais abrangentes na forma de gerir e liderar, bem como na governança em todos os níveis da organização (Gurd \& Ifandoudas, 2014; Gandomani \& Nafchi, 2015; Dikert,
Paasivaara, \& Lassenius, 2016; Hoda \& Noble, 2017; Moe \& Dingsøyr, 2017; Paterek, 2018).

Cabe aqui destacar que o processo de transição é complexo e longo, limitado por diversas barreiras e desafios únicos. Além disso, esse processo de mudança requer, portanto, um esforço substancial em termos de custo e recursos de longo prazo, bem como engajamento e cooperação entre diferentes unidades de negócios da organização do projeto evolucionário (Paterek, 2018). A mudança para o modelo ágil é um momento de transição pelo fato de ser necessário ajustar processos, cultura, ferramentas e uma gestão de projetos adaptativa, híbrida, permitindo a convivência temporária dos dois modelos (Adelakun et al., 2017; Fernandez \& Fernandez, 2008).

\subsection{Métodos Híbridos}

A necessidade de abordagens híbridas de gerenciamento de projetos, combinando práticas tradicionais e ágeis já se fazia sentir em 2003, pouco depois do Manifesto Ágil, (Fernandez e Fernandez, 2008). De fato, apesar das vantagens percebidas, já se observava o surgimento de diversos desafios gerenciais na adoção de práticas ágeis. Em alguns ambientes, por exemplo, elas não se adequavam facilmente, sugerindo que as características de adaptabilidade poderiam ser complementadas com práticas tradicionais, mais estruturadas (Adelakun et al., 2017; Fernandez \& Fernandez, 2008). 
Para se adequar a essa realidade, muitas organizações decidiram adotar as práticas ágeis sem abandonar as tradicionais, combinando diferentes metodologias (Vinekar et al., 2006). Dessa forma, passaram a usar os métodos híbridos para responder ao desafio das mudanças constantes de requisitos, procurando aproveitar tanto a visão adaptativa das abordagens ágeis quanto a solidez dos métodos tradicionais (Adelakun et al., 2017).

As práticas dos métodos ágeis são então adaptadas às dos métodos tradicionais adequando-se as necessidades da organização e equilibrando as vantagens e desvantagens de cada método (Kusters et al., 2017).

Para algumas organizações, mostrouse ser mais benéfico mudar inicialmente de uma abordagem não-ágil para uma abordagem híbrida e, em seguida, para uma mentalidade totalmente ágil por meio da crescente adoção de todos os seus princípios (Gill et al., 2018). A esse respeito, Burman (2015) reporta que a realidade da adoção de práticas ágeis, não se dá pela utilização completa da metodologia, mas de uma forma híbrida, com áreas da organização que permanecem atuando dentro de outras escolas e filosofias metodológicas.

Analisando os ambientes híbridos de projeto, Kusters et al. (2017) observam a existência de problemas relacionados às diferenças entre as abordagens usadas. Gill et al. (2018) afirmam que num cenário de integração, para a construção de uma metodologia adaptativa híbrida, surge o desafio de determinar os elementos relevantes do processo e como adaptá-los às práticas ágeis. Por sua vez, Cram e Marabelli (2018) ressaltam a dificuldade de se chegar a um ponto em que a fusão de técnicas ágeis e tradicionais possa funcionar de maneira eficaz. Apontam, ainda, que esse processo se torna particularmente difícil quando as equipes foram treinadas apenas na condução de projetos no formato tradicional.

\section{Método}

Este artigo está estruturado sob a abordagem qualitativa descritiva e utilizou como estratégia de pesquisa o estudo de caso único descritivo. De acordo com Kvale (2008) a pesquisa qualitativa é apropriada quando se pretende abordar fenômenos sociais a partir do ambiente real de trabalho, analisando experiências relacionadas às práticas profissionais dos indivíduos. Nas pesquisas qualitativas, o estudo de caso único permite que as informações sejam trabalhadas de forma precisa, sintética, sistemática e alinhada com um fenômeno a ser estudado (Patton, 2005). Os pesquisadores apoiam-se em sua experiência para refletir e tomar decisões durante $o$ processo de produção do conhecimento, conforme sugere Flick (2014).

De acordo com Creswell e Poth (2016) e Yin (2015), os estudos de caso são apropriados para examinar situações da vida real contemporânea em ambientes nos quais o pesquisador tem pouco ou nenhum controle. Em vista disso, deve-se apoiar em uma profunda e detalhada coleta de dados, que 
envolva fontes de informação diversas como observações, entrevistas, material audiovisual, documentos e relatórios. Por sua vez, da Silva et al. (2017) acrescentam que a importância dos estudos de caso decorre das informações que apresentam sobre fenômenos pouco estudados. Esse tipo de estudo facilita as pesquisas que buscam descrever e explicar fenômenos sociais complexos, como os processos organizacionais (Ponelis, 2015).

Um estudo de caso descritivo é aquele que provê uma descrição exaustiva do fenômeno dentro do contexto real em que ele ocorreu, contribuindo com a compreensão de acontecimentos, centrando-se nas questões "Como" e "Porquê" (Yin, 2015). Nesse sentido, o mesmo autor considera que o estudo de caso único se justifica quando se trata de um caso representativo das circunstâncias e condições da situação que se deseja captar. Assim, presume-se que o aprendizado adquirido numa empresa típica é informativo sobre outras empresas da mesma indústria.

\subsection{A empresa}

Esta pesquisa foi realizada em uma empresa brasileira de grande porte, do ramo de Telecom, com mais de mil funcionários, sediada na cidade de São Paulo, que está vivenciando um processo de transformação ágil. Trata-se de uma mudança estratégica para adequar a empresa a uma nova realidade de mercado, na qual são necessários processos mais ágeis, entregas de valor aos clientes e obtenção de vantagem competitiva em relação à concorrência. A empresa foi escolhida pelo fato de permitir a oportunidade de analisar a transição de uma abordagem tradicional para uma abordagem ágil em projetos de infraestrutura de TI. A passagem dessa área e suas equipes por um contexto de Transformação Ágil compreende um desafio digno de estudo, pois abrange uma mudança de cultura, processos e modelo de negócios, em times com habilidades e especialidades técnicas distintas.

\subsection{A coleta de dados}

Neste trabalho, foram coletados e analisados documentos eletrônicos referentes às características da empresa, institucionais de projetos, documentos com a proposição de transformação ágil (estratégia, processos e cultura), bem como documentos e processos específicos para os projetos de infraestrutura de TI. No contexto tradicional, foram analisados: solicitação de estudos de projetos, escopo, status report de projetos, cronograma, relatório de portfólio de projetos, atas de reuniões, planos de comunicações, documentos de entrega de fase, encerramento do projeto e solicitação de mudança. No contexto ágil, foram analisados: planilha de projetos no formato de quadro kanban (representando as fases de entregas dos projetos), status report com visão ágil e tradicional mescladas, documento de medição do nível de maturidade, planilha relativa aos indicadores do portfólio de projetos com medições difundidas para os modelos e documentos que explicavam a 
proposição da adoção dos métodos ágeis e a estratégia de implantação.

As entrevistas semiestruturadas constituem um formato flexível, acessível e inteligível, permitindo que os entrevistados expressem suas ideias de maneira espontânea, facilitando o entendimento de como percebem suas atividades e seu ambiente de trabalho $(\mathrm{Qu}$ \& Dumay, 2011). A realização das entrevistas deste estudo de caso seguiu as quatro diretrizes iniciais do processo sistemático proposto por Kvale (2008): i) formulação do objetivo e do tema; ii) planejamento; iii) realização das entrevistas; iv) transcrição. Nesse sentido, o tema refere-se à implantação de um processo de transformação ágil em uma empresa de Telecom e o objetivo refere-se à descrição de um momento específico de transição da empresa. $\mathrm{O}$ perfil dos entrevistados foi definido, o protocolo de entrevista foi elaborado e, o formulário de consentimento informado foi criado.

As perguntas constantes no protocolo de entrevista abrangem os aspectos do processo de transformação ágil considerados relevantes para responder à questão de pesquisa, de acordo com a revisão de literatura e os objetivos da pesquisa: i) motivação e preparação do processo de implantação de métodos ágeis; ii) atuação da alta gerência no processo de implantação; iii e iv) relação dos entrevistados com as metodologias tradicionais e ágeis, abrangendo o conhecimento, a experiência e a opinião sobre cada uma; v) a relação dos entrevistados com o modelo híbrido; e vi) estágio de aplicação dos processos ágeis nos projetos da empresa, seus facilitadores e barreiras. Para incentivar a discussão abrangente, foram elaboradas perguntas abertas, fundamentadas na literatura e nos objetivos da entrevista. A Tabela 1 apresenta o perfil dos entrevistados. 
Silveira, T. B. J., \& Lemos, D. da C. (2021, Ed. Esp. jan./abr.). Colaboração com clientes e inovação aberta no desenvolvimento àgil de software: uma revisão sistemática de literatura

Tabela 1 - Perfil dos entrevistados

\begin{tabular}{|c|c|c|c|c|c|}
\hline Id & Cargo & Departamento & Objetivo & $\begin{array}{l}\text { Envolvimento com a } \\
\text { organização }\end{array}$ & $\begin{array}{l}\text { Experiência } \\
\text { em projetos }\end{array}$ \\
\hline E01 & $\begin{array}{c}\text { Gerente de } \\
\text { projetos de } \\
\text { infraestrutura de } \\
\text { TI }\end{array}$ & $\begin{array}{l}\text { Infraestrutura } \\
\text { de TI }\end{array}$ & $\begin{array}{l}\text { Entregar e acompanhar os } \\
\text { projetos que envolvem } \\
\text { sistemas ou infraestrutura }\end{array}$ & $\begin{array}{c}\text { Terceirizado, alocado } \\
\text { em tempo integral na } \\
\text { organização }\end{array}$ & 15 anos \\
\hline E02 & $\begin{array}{c}\text { Pré-vendas e } \\
\text { Arquiteto de } \\
\text { soluções de } \\
\text { infraestrutura de } \\
\text { TI }\end{array}$ & $\begin{array}{l}\text { Infraestrutura } \\
\text { de TI }\end{array}$ & $\begin{array}{l}\text { Soluções para atender das } \\
\text { empresas do grupo, nos } \\
\text { diversos países que atuam }\end{array}$ & $\begin{array}{c}\text { Terceirizado, alocado } \\
\text { em tempo integral na } \\
\text { organização }\end{array}$ & 20 anos \\
\hline E03 & $\begin{array}{c}\text { Gerente de } \\
\text { projetos de } \\
\text { infraestrutura de } \\
\text { TI }\end{array}$ & $\begin{array}{l}\text { Infraestrutura } \\
\text { de TI }\end{array}$ & $\begin{array}{l}\text { Projetos de migração de } \\
\text { bases, dados e aplicações } \\
\text { para uma nova } \\
\text { infraestrutura }\end{array}$ & $\begin{array}{c}\text { Terceirizado, alocado } \\
\text { em tempo integral na } \\
\text { organização }\end{array}$ & 13 anos \\
\hline E04 & $\begin{array}{c}\text { Gerente de } \\
\text { projetos de } \\
\text { infraestrutura de } \\
\text { TI }\end{array}$ & $\begin{array}{l}\text { Infraestrutura } \\
\text { de TI }\end{array}$ & $\begin{array}{c}\text { Migração de bancos de } \\
\text { dados para ambientes em } \\
\text { nuvem. }\end{array}$ & $\begin{array}{c}\text { Terceirizado, alocado } \\
\text { em tempo integral na } \\
\text { organização }\end{array}$ & 17 anos \\
\hline E05 & $\begin{array}{c}\text { Gerente de } \\
\text { projetos de } \\
\text { infraestrutura de } \\
\text { TI }\end{array}$ & $\begin{array}{l}\text { Infraestrutura } \\
\text { de TI }\end{array}$ & $\begin{array}{c}\text { Projetos de infraestrutura: } \\
\text { para atender negócios, } \\
\text { unificação e migração de } \\
\text { sistemas para ambientes em } \\
\text { nuvem }\end{array}$ & $\begin{array}{c}\text { Terceirizado, alocado } \\
\text { em tempo integral na } \\
\text { organização }\end{array}$ & 12 anos \\
\hline
\end{tabular}

Fonte: Elaborada pelos autores.

Foram entrevistados quatro gerentes de projeto e um arquiteto de infraestrutura de TI que atuam na empresa estudada. Todos foram convidados pessoalmente e informados sobre o objetivo, o tema e a duração estimada da entrevista. A participação foi voluntária e as entrevistas foram agendadas sempre de acordo com a disponibilidade e a preferência dos entrevistados. Todas as entrevistas foram presenciais e tiveram duração média de 60 minutos. Os entrevistados foram informados sobre a garantia de privacidade e confidencialidade, assim como receberam uma breve explicação sobre o tema e os objetivos da entrevista. As entrevistas foram gravadas e transcritas passando o discurso oral para o texto escrito, utilizando-se o software Audipo no apoio à transcrição. Para garantir a fidelidade, $o$ texto final transcrito foi comparado às gravações e corrigido quando necessário.

Em estudos de caso, a técnica da observação é frequentemente combinada com as entrevistas (Godoy et al., 2017). A observação participante é uma técnica que pode ser usada para coleta de dados em grandes organizações, proporcionando oportunidade de acesso a eventos que de outra forma não seria possível, captando a realidade do ponto de vista de um observador interno ao caso (Yin, 2015). O pesquisador passa a ser mais que um simples espectador, podendo participar dos eventos que estão sendo estudados. Neste trabalho, a observação participante foi realizada por um dos autores, que participou ativamente do processo de 
transformação ágil que está sendo estudado (Godoy et al., 2017).

A observação participante ocorreu entre os meses de julho de 2019 e março de 2020. Nesse período, o pesquisador participou de todo o processo de implantação dos métodos ágeis na equipe de Infraestrutura de TI, bem como da adoção de práticas que buscavam otimizar as atividades diárias das equipes e trazer mais valor para o negócio. $\mathrm{O}$ pesquisador também participou das reuniões e discussões para a formação do time e de outras para definição dos procedimentos, métodos, artefatos e relatórios necessários para o novo modelo de gestão. Essa fase de preparação e conscientização do time envolveu a conceituação do que é agilidade, os objetivos estratégicos e o direcionamento adotado para a transformação ágil da organização.

A vantagem mais importante da utilização de fontes múltiplas de evidência é o desenvolvimento de linhas convergentes de investigação, possibilitando o processo de triangulação de dados (Yin, 2015). A triangulação é uma estratégia de validação que combina metodologias, obtendo dados sobre o mesmo acontecimento a partir de duas ou mais fontes, tornando mais convincentes e acuradas as descobertas ou conclusões, aumentando a confiabilidade dos resultados (Meirinhos \& Osório, 2016). Neste trabalho, a validação dos dados por triangulação deu-se mediante informações obtidas nas análises de documentos, nas análises do conteúdo das entrevistas e na observação participativa.

\subsection{A análise dos dados}

Em consonância com o processo delineado para esta pesquisa, a análise do conteúdo das entrevistas foi realizada por meio da técnica proposta por Bardin (2011). O software ATLAS.ti, versão 7.5.4, foi utilizado como suporte na análise para automatizar o agrupamento das falas, armazenar textos e resultados. A técnica utilizada compreende três fases: i) pré-análise, na qual aconteceu a leitura geral do material transcrito e decidiu-se pelo agrupamento das falas de cada entrevistado relacionadas aos seis temas definidos na fase de planejamento do protocolo; ii) exploração do material coletado, que foi recortado e agrupado por tema; e iii) tratamento dos resultados, inferência e interpretação, fase na qual os conteúdos manifestos e latentes do material coletado e categorizado são interpretados com o respaldo do referencial teórico.

A análise do conteúdo das entrevistas e sua comparação com os dados da observação participante permitiram em primeiro lugar a compreensão do contexto em que ocorre o processo de transformação ágil, na ótica de participantes envolvidos. Adicionalmente, forneceram a visão da sequência dos acontecimentos, na maneira como foram por eles percebidos, permitindo descrever e elaborar um modelo da situação em que se encontrava o processo quando a pesquisa foi conduzida. Durante este processo, a análise concomitante dos documentos possibilitou a comparação das características dos modelos 
tradicional e ágil sendo aplicados, bem como a avaliação do impacto das mudanças, em andamento na empresa.

\section{Resultados e discussão}

Nesta seção apresentamos os resultados da pesquisa. Inicialmente, descrevemos a análise do material coletado nas entrevistas, documentos e observação participante. Em seguida, apresentamos o framework híbrido de gerenciamento de projetos identificado e proposto para os processos sendo seguidos na transformação ágil, a partir do conhecimento adquirido durante a análise realizada.

\subsection{Motivação e preparação do processo de implantação}

Conforme foi observado pelo pesquisador e pelos entrevistados, o processo iniciou-se com o patrocínio da alta administração. Esta informou que o objetivo da transformação ágil é levar os conceitos de agilidade não apenas para os projetos, mas para todas as áreas da empresa, dentro das características de cada uma. Com a iniciativa, a diretoria espera trazer maior agilidade aos processos, melhorar o tempo de resposta às necessidades de mercado e agregar maior valor para os clientes e para a organização.As áreas de TI foram escolhidas para iniciar o processo de mudança organizacional. Nesse cenário, encontra-se a Infraestrutura de TI, uma área complexa, onde as equipes são responsáveis pelo data center, conectividade e redes, servidores, computação distribuída, cloud computing, backup, banco de dados, storage, operação e projetos. Os entrevistados demostraram conhecimento dos objetivos da alta gestão. Entre os resultados positivos esperados foram citados rapidez, agilidade nas entregas, transparência, maior interação entre as pessoas, aumento da competitividade e melhoria do posicionamento no mercado.

No início do processo, uma área foi preparada para cuidar da transformação ágil. Seus integrantes foram treinados para atuar como agile coaches para disseminar conhecimento e treinar equipes. Uma empresa de consultoria foi contratada, disponibilizando profissionais certificados e experientes, para auxiliar e orientar nas etapas de adequação e mudança. Com esse apoio, foi criado o roadmap de transformação. A diretoria determinou que a transição não afetasse os projetos em andamento, em sua maioria conduzidos pelos métodos tradicionais.

Observou-se, porém, que os agile coaches começaram a atuar sem o treinamento e preparo devido das equipes. $\mathrm{Na}$ área de infraestrutura de TI, tal ação causou resistência por parte dos gerentes de projeto, receosos de serem cobrados por coisas sobre as quais não detinham um conhecimento adequado. A despeito desse fato, o processo prosseguiu. A diretoria interveio, ratificando que a mudança era a nível organizacional e que os resultados dos projetos seriam avaliados com base na abordagem ágil. Solicitou ainda o apoio e a participação de todos para atuar dentro do 
novo paradigma. Foi reforçado que os times seriam autogerenciados.

Durante essa fase de aprendizado e aculturamento, o pesquisador observou vários ajustes e tratativas para realizar a migração para o novo modelo, em que foram definidos os cerimoniais do ágil, os papéis para a condução das atividades e as agendas das reuniões. Segundo ele, percebeu-se aí que tal transformação é um processo que demanda ajustes gradativos e constantes. Dentro desse cenário, parte dos documentos e controles foram ajustados à realidade que se buscava $\mathrm{e}$ outra parte foi mantida, respectivamente para as fases do processo em que os métodos ágeis seriam utilizados e para as outras em que não havia como usá-los.

Nas respostas sobre a preparação das pessoas, entretanto, os entrevistados não apontaram diretamente os problemas relacionados à insuficiência de treinamento. Todos mencionaram um mesmo conjunto básico de técnicas e ações: workshops, reuniões de divulgação e palestras com profissionais especializados. Em consonância com o observado, E05 relatou que a empresa "não mudou todos os projetos pra metodologia ágil, (...) começou a preparar alguns projetos para uma entrega mais adiantada e contínua das atividades, e (...) a colocar ritmo em alguns projetos". E04 mencionou que seria necessária uma "estratégia de difundir $e$ promover a metodologia ágil, considerando uma certa flexibilidade diante da dificuldade de cada área".
Apenas E01 comentou a respeito do caminho da transição por meio da cobrança da diretoria por entregas rápidas. Os projetos estariam usando cada vez mais "um pouquinho do tradicional e um pouquinho do, do ágil" e essa pressão seria capaz de acelerar o uso da metodologia ágil. Ainda assim, ele ressaltou que "em algumas fases não vai dar para fugir muito do tradicional".

\subsection{Atuação da alta gerência no processo de implantação}

O entrevistado E03 resumiu a participação da alta gerência, afirmando que "ela foi envolvida porque toda a organização precisou ser envolvida e está sendo envolvida". Todos os entrevistados, menos um, responderam que os gestores atuam bastante, no sentido de "buscar que todos tenham engajamento" (E01), "facilitando para todos o acesso ao conhecimento, para que todos se desenvolvam e gerem melhores resultados" (E02), "buscando e recebendo informações" (E02 e E03), para que "diretoria, gestores e outras equipes estejam realmente na mesma página" (E03), "todos no mesmo time" (E02). O gerente de projetos E04 acrescentou que isso precisava acontecer, pois " $a$ empresa requer da diretoria alta performance (...) $e$ $100 \%$ de engajamento e envolvimento", entendendo que " $a$ alta gerência $d a$ companhia (...) está engajada em promover o modelo ágil".

Apenas o entrevistado E05 relatou ainda não sentir a atuação da alta gerência com 
projetos ágeis em sua área. Não obstante, relatou ter observado "algumas áreas específicas de negócios, (...) que têm o interesse nesse progresso e agitaram essas medidas (...) através de metodologia ágil", reforçando a ideia da estratégia de implantação gradual. Nesse sentido, E02 observa que há um entendimento de que a alta gerência deu início ela própria ao processo, "lá em cima (...), para ser implantado de cima para baixo", o que impulsionou "algo muito bom que fez muita gente reaprender e ter vários estímulos de trabalho, trouxe um fôlego novo para muita gente". Concordando, E03 opina que a alta gerência buscou trilhar o caminho de "uma implementação com mais eficiência e com mais certeza que dará certo".

Apesar da visão positiva sobre os esforços da diretoria, E01 observou que existe "um certo distanciamento de algumas áreas $e$ de algumas pessoas". Ele credita que esse comportamento é relacionado ao fato de o processo ser "uma coisa nova e às vezes as pessoas não estão ainda entendendo qual seria o ganho para eles". Acrescenta, porém, que "todos têm que entrar nesse processo (...) recente e as companhias levam um tempo para se adaptar".

\subsection{Relação dos entrevistados com as metodologias tradicionais}

Todos os entrevistados declararam ter formação e conhecimento significativo das metodologias tradicionais, sendo que o E04 é certificado pelo Project Management Institute,
PMI. Em consequência, a experiência profissional do grupo está fortemente ligada a esses modelos, padrões e práticas, sendo que os entrevistados E01, E02 e E04 declararam ter trabalhado prioritária ou exclusivamente com métodos tradicionais desde o início da carreira. Essa situação remete ao estudo de Cram e Marabelli (2018) que ressaltam as dificuldades em um processo de transformação ágil em que as equipes têm ampla experiência na aplicação dos modelos tradicionais. Apesar disso, os entrevistados não demonstraram uma percepção fechada e inflexível em favor dos métodos tradicionais, reconhecendo o valor de outras práticas. E01 e E02 observaram que os modelos tradicionais já estão enraizados para a execução de projetos como infraestrutura de TI e construção civil, mas que os métodos ágeis podem ser mais adequados para desenvolvimento de software, de produtos, e outros projetos de curta duração.

Avaliando o gerenciamento tradicional, E03 o vê "bastante engessado" e supõe que muitos praticantes compartilham essa visão. E04 o considera mais conservador, mas percebe existir uma adaptação dos projetos ao mercado, influenciada pelas exigências da legislação e ainda identifica uma evolução nos modelos e na forma de trabalho, no sentido de propiciar maior flexibilização. E05 o considera eficaz, mas defende sua aplicação customizada, de acordo com a cultura, a história e os processos da empresa, não seguindo rigidamente os manuais. 


\subsection{Relação dos entrevistados com as metodologias ágeis}

No que se refere aos métodos ágeis, o grau de conhecimento dos entrevistados é variado, porém todos relataram ter pouca ou nenhuma experiência em utilizá-los. E01 e E05 apenas assistiram a alguns workshops e E02 estudou bastante por conta própria. Em contraposição, E03 e E04 têm conhecimento sobre o assunto, são ambos certificados como Scrum Master, sendo que o primeiro é também certificado como Agile Coach. Quanto à experiência, E04 já atuou com projetos ágeis de implantação de sistemas. No entanto, E01 e E02, E04 e E05 disseram que só agora estão conhecendo mais, aprendendo na prática, com o suporte dos técnicos da consultoria. Nesse sentido, os trabalhos de Kusters et al. (2017) e Cram e Marabelli (2018) fazem referência à lacuna teórica que existe nas pesquisas sobre transformação ágil num momento de transição, salientando sua relevância para os profissionais envolvidos, particularmente aqueles com pouca experiência nas práticas ágeis.

Em relação às características, benefícios e barreiras para a sua aplicação nos projetos que gerenciam, os entrevistados E01 e E02 ressaltaram a interação e a colaboração entre as equipes que trabalham nos projetos. E02 e E03 destacam que os benefícios e as entregas de valor são mais rápidos e constantes. E02 ainda lembrou a rapidez nas mudanças, o controle do tempo, das tarefas e das alocações de pessoal, resumindo que o ágil "tem dominado toda a (área de) infraestrutura,
(...) é um caminho que está consolidado (...) é um processo que veio para ficar".

E04 e E05 entendem que os métodos ágeis são mais eficientes em projetos em que se exige mais criatividade, como o desenvolvimento de software e de produtos. Para E04, são necessárias adaptações para aplicá-los em outras áreas como telecomunicações e contabilidade. Dessa forma, veem complexidade e menos eficácia na implementação ágil de produtos de prateleira, prontos para instalação, como os da área de infraestrutura de TI, que "naturalmente (...) já vêm com as boas práticas de como (...) gerenciar um projeto de implantação", conforme disse o entrevistado E04.

\subsection{Relação dos entrevistados com o modelo híbrido}

Todos os entrevistados apontaram que o momento da transição é de coexistência positiva entre os dois modelos, tradicional e ágil. E03, por exemplo, vê a empresa usando um método híbrido, pois "vários elementos (...) fazem parte do processo tradicional, só que aplicado muito mais rapidamente, (...) há uma interação, uma conexão mais rápida. (...) Esse formato dá certo, então é válido". E01 e E02 observaram que a mescla entre tradicional e ágil é uma evolução, já faz parte do cotidiano e cada cenário tem suas peculiaridades, suas mudanças, seus benefícios e suas fraquezas.

Em vista disso, declarou E01 "a gente vai ainda ter que conviver muito, por muito tempo num modelo híbrido". E02 também 
pensa que "é assim que as empresas vão tratar daqui para frente, (...) elas ainda têm esse mix", até surgir uma metodologia consolidada. E04 apoia o aproveitamento das vantagens dos modelos híbridos, pois se "existe uma metodologia que (...) propõe (...) mesclar um projeto ágil com tradicional, (...) alguém lá atrás já passou um sufoco querendo fazer só ágil ou só tradicional, até que (...) falou: "vamos mesclar que vai dar certo",. Segundo E05, com essa mescla pode-se "ganhar até mais agilidade, fazer o processo se mover de maneira mais rápida (...) pra conseguir soluções mais rápidas".
4.6 Estágios de aplicação dos processos ágeis, facilitadores e barreiras

A partir das respostas dos entrevistados, foram identificados quatro estágios de aplicação das práticas ágeis nos processos dos projetos de infraestrutura da empresa, confirmando que a transformação ágil está acontecendo de forma gradual e individualizada. Os estágios encontrados foram denominados: i) sem aplicação; ii) em preparação; iii) em adaptação; e iv) em aplicação plena, destacados na Figura 1.

\section{\begin{tabular}{|l|l|l|l|l|l|l|l|l|l|}
\hline Sem Aplicação & Em Preparação & Em Adaptação & Em Aplicação \\
\hline
\end{tabular}}

Figura 1 - Estágios de aplicação dos métodos ágeis na empresa

Fonte: Elaborada pelos autores

No primeiro estágio, "Sem aplicação", conforme explicado por E05, os processos dos projetos executados ainda são realizados pela metodologia tradicional seguindo uma sequência padronizada de passos e produzindo documentos preconizados. São aplicados os processos de gestão de mudança em produção, com aberturas de chamados e workflow de aprovações.

Observando as outras áreas, E05 identificou que na implantação da sua área os processos ágeis serão mais apropriados para substituir ou complementar os tradicionais, na parte de execução. Na fase de iniciação, diz ele, a natureza dos projetos ainda requer documentação abrangente e atividades bem definidas. Adicionalmente, E05 sugere aumentar a rapidez reduzindo a dependência das datas de entrega e dos fluxos internos de aprovação, possibilidade também percebida durante a observação participante. Ele não vê, hoje, "essa possibilidade de atuar como ágil, sendo que a empresa está amarrada nesses processos". Idealmente, segundo ele, haveria uma equipe de infraestrutura subordinada ao gerente de projeto, fazendo as implantações em ambiente de produção ao final do projeto, com apenas uma requisição.

Pode-se perceber nessa situação a importância da integração e colaboração entre as implantações dos métodos ágeis nas diversas áreas envolvidas na execução de um 
mesmo processo, como sugerido por Paterek (2018). Processos lentos transmitem sua lentidão aos que deles dependem, prejudicando a agilidade.

No segundo estágio, "Em preparação", foi evidenciado que em consonância com a estratégia de implantação gradual e individualizada, o projeto mais recente de E03 ainda está sendo executado no formato tradicional, embora se trate de uma tecnologia nova. No entanto, ele já está preparando a transição em sua área, desenvolvendo uma proposta de processo ágil de trabalho, para aprovação da diretoria e aplicação em pequenos projetos piloto, para avaliação.

Esse gerente de projetos acha que o facilitador mais importante na passagem para $\mathrm{o}$ ágil foi a distribuição igual do conhecimento para todas as camadas e não vê barreiras que impeçam a realização dos projetos no modelo ágil. Ressalta que essa metodologia já está sendo aplicada em áreas que interagem com seus projetos, em processos de demandas e medições.

No terceiro estágio, "Em adaptação", os entrevistados E01 e E04 atuam em projetos em que a aplicação de métodos ágeis já foi iniciada passando agora por um movimento natural de adaptação aos novos processos. O primeiro entrevistado mencionou estar recebendo suporte da consultoria e considera isso um facilitador, aliado ao conhecimento que já possuía da metodologia ágil. E01 relata que a adaptação está acontecendo vagarosamente e ainda existem dúvidas quanto à aplicação da metodologia, o que é certo, errado, obrigatório ou recomendação. A periodicidade das reuniões diárias foi alterada para semanal. Estão sendo aplicados os processos de retrospectiva, plannings, sprints, desenvolvimento e conhecimento das histórias. Seu projeto está sendo trabalhado em pequenas entregas, com o planejamento e a execução acontecendo várias vezes, o que também foi registrado na observação participante.

Como exemplo, E04 cita um projeto de migração de 300 bancos de dados que teve seu cronograma dividido em 10 fases. Ao invés da rígida sequência de implantação anterior, definida no início do projeto, a priorização dos bancos e a montagem do cronograma acontecem agora no início de cada fase, considerando respostas a mudanças no mercado e na empresa.

A falta de experiência com os novos processos e a forte cultura do uso do modelo tradicional são barreiras na passagem para o ágil, identificadas por E01. Ele percebe dificuldades, por exemplo, na forma de reportar as entregas e mostrar os resultados. Nesse sentido, relata que os diretores preferem o relatório de status no formato antigo, em vez de receber a visão ágil da evolução do projeto, extraída da plataforma de gestão, utilizando a ferramenta de gestão de projetos denominada Jira.

O gerente E04, por sua vez, relatou que a maioria dos novos projetos de infraestrutura de TI está sofrendo adaptações para funcionar no modelo ágil. Os projetos em 
andamento no modelo tradicional, entretanto, continuam sendo executados da mesma forma. Comparando com os processos tradicionais, E04 considera o ágil mais adequado e eficiente nas fases de execução e encerramento e mais difícil de implantar nas fases de iniciação e de planejamento.

No processo de transição ágil, E04 acha que "o primeiro fator mais importante que facilita (...) uma troca de modelo de gerenciamento de projeto é a disposição do time (...) em mudar", assim como "o segundo princípio (...), é a disposição da empresa em fazer isso acontecer". Segundo ele, isso reflete o princípio ágil de valorizar as pessoas sobre os processos e ferramentas. E04, acrescenta que, em geral, os profissionais atentos à evolução do mercado já vêm se preparando. "No início (...), alguns até trazem sugestões sobre como fazer a transição", comenta.

No que se refere às barreiras para a implantação de processos ágeis, E04 diz que a maior delas é "não observar quando um projeto não pode ser $100 \%$ ágil". Ele acredita que ao decidir fazer a transição, os gestores devem analisar cada área envolvida, observando se tem condições de alcançar o nível de absorção e aplicação da metodologia ágil que se espera dela. Nesse sentido, E04 vê que nem todos os projetos de infraestrutura de TI conseguem ser $100 \%$ ágeis, uma vez que neles trabalha-se com produtos de mercado, com instruções definidas de implantação e utilização. Na implantação de datacenters, explica, é necessário seguir o plano recomendado pelo fornecedor, não há espaço para responder a eventuais mudanças. Segundo ele, "na infra, paralelizar, modificar, ajustar, são ações que você tem pouca dirigibilidade, (...) não consegue modificar a rota, (...) tem que fazer daquela forma e pronto".

No quarto estágio, "Em aplicação plena", o arquiteto de soluções E02 relata que hoje "praticamente todos os projetos em que eu trabalho já estão na metodologia ágil". Toda a parte de gestão, controle e implementação estão implantados, de forma a interagir com as outras equipes e realizar as entregas usando a metodologia. Segundo ele, as ferramentas visuais de controle, como o quadro Kanban, e o planejamento em iterações foram os maiores facilitadores da transição. Os benefícios desses dois fatores também foram identificados na observação participante.

O entrevistado E02 ressaltou, porém que "existem vários processos da metodologia tradicional que não podem ser substituídos", citando toda a parte de viabilidade e iniciação do projeto, análise de custos, alocação de recursos, formalização, alocação dos gerentes, validação do escopo, acordos com os gestores e a parte de conclusão do projeto. "Esses processos não mudaram", observou.

Corroborando a percepção do gerente E05 e as conclusões da observação participante, E02 considera os processos seguidos pelo suporte de TI como uma barreira. Chamados, respostas, entregas e manutenção do ambiente em operação são processos já enraizados, dificultando a 
Gonçalves, M. L. do A., Silva, R. A. C. da, Silva, E. A. C., \& Penha, R. (2021, Ed. Esp. jan./abr.). Processo de transformação ágil em uma empresa brasileira de Telecom

mudança para processos ágeis. Nesse contexto, uma falha operacional precisa ser sempre respondida da mesma maneira, para colocar de volta o ambiente no ar, por exemplo.

E02 ainda cita os processos do setor público como uma barreira para a implantação dos métodos ágeis nos projetos da empresa junto ao governo, como a construção de usinas hidrelétricas e barragens. São projetos longos, que dependem de verbas e de metas bem definidas, detalhadas em planejamentos governamentais de anos anteriores. A quebra desse mecanismo é difícil, exigindo uma mudança muito grande em várias esferas de governo.

\begin{tabular}{|c|c|}
\hline & Facilitadores \\
\hline \multirow{6}{*}{ Gerenciais } & Envolvimento e apoio da alta gestão ao processo de implantação \\
\hline & Estratégia de implantação em âmbito geral \\
\hline & Estratégia de implantação gradual e individualizada \\
\hline & Distribuição equitativa de conhecimento para todas as áreas \\
\hline & Contratação de uma consultoria especializada \\
\hline & Divulgação maciça do projeto em todos os canais de comunicação da empresa \\
\hline \multirow{3}{*}{ Culturais } & Consciência da equipe sobre a passagem para o ágil como tendência \\
\hline & Conhecimento prévio de alguns gerentes, apoiados pelos treinamentos \\
\hline & Disposição da equipe para realizar a mudança \\
\hline \multirow{4}{*}{ Operacionais } & Divulgação e adoção das boas práticas surgidas nas diversas áreas \\
\hline & Planejamento e execução das atividades em iterações \\
\hline & Adoção do conceito de pequenas entregas, do mínimo produto viável (MVP) \\
\hline & Utilização de ferramentas visuais de controle \\
\hline \multicolumn{2}{|r|}{ Barreiras } \\
\hline Gerenciais & Falta de entendimento da direção sobre o os limites de implantação nas áreas \\
\hline \multirow{4}{*}{ Organizacionais } & Processos internos lentos de aprovação de mudanças pelo suporte de TI \\
\hline & Equipes de suporte não subordinadas aos gerentes de projeto \\
\hline & Equipes de suporte cuidam dos projetos e das atividades permanentes \\
\hline & Dependência das datas de entrega na implantação \\
\hline \multirow{2}{*}{ Operacionais } & Planos de implantação definidos pelos fornecedores, sem espaço para mudança \\
\hline & Necessidade de atividades definidas na fase de iniciação \\
\hline
\end{tabular}


Gonçalves, M. L. do A., Silva, R. A. C. da, Silva, E. A. C., \& Penha, R. (2021, Ed. Esp. jan./abr.). Processo de transformação ágil em uma empresa brasileira de Telecom

\begin{tabular}{|c|l|}
\hline \multirow{2}{*}{ Culturais } & Necessidade de documentação abrangente na fase de iniciação \\
\cline { 2 - 2 } & Alguns processos da metodologia tradicional não substituíveis por ágeis \\
\hline \multirow{2}{*}{ Legais } & Cultura enraizada do uso do modelo tradicional \\
\hline & Exigências legais nos projetos da área governamental na aplicação dos processos da metodologia ágil \\
\hline
\end{tabular}

Figura 2 - Facilitadores e barreiras à implantação dos processos ágeis.

Fonte: Elaborada pelos autores

$\mathrm{Na}$ Figura 2 são apresentados os fatores considerados como facilitadores ou barreiras à implantação dos processos da metodologia ágil hoje em curso na empresa, de acordo com as respostas dos entrevistados. Os fatores foram categorizados em gerenciais, organizacionais, operacionais, culturais e legais, segundo sua natureza. As diversas atribuições e a independência da área de suporte de TI, bem como os processos obrigatórios da gestão de mudança determinam as datas de entrega, demandando um planejamento extensivo anterior à fase de execução e transferindo a dependência de datas para a conclusão das atividades e o encerramento do projeto. Os produtos a serem implantados, como resultado dos projetos têm regras de instalação fixas, definidas pelos fornecedores, impedindo mudanças que tragam mais flexibilidade. A natureza dos produtos a serem entregues também exige estudos de viabilidade cujos processos são fortemente adequados ao modelo tradicional de gerenciamento de projetos.

No que se refere aos processos da metodologia ágil, fatores gerenciais, operacionais e culturais facilitaram sua aplicação nas fases de execução e implantação dos projetos, fases em que os produtos e serviços são efetivamente desenvolvidos. Apoiadas pelo conhecimento adquirido nas capacitações e pela atuação da consultoria, as equipes definiram e implantaram processos ágeis de trabalho nessas fases. Isso reflete as afirmações dos entrevistados que apenas na fase de execução dos projetos é possível quebrar as entregas em partes menores, dandolhes visibilidade e possibilitando ações rápidas para acertos e modificações na entrega do produto.

\subsection{Framework híbrido proposto para a situação da transição}

A análise das respostas dos entrevistados e da observação participante permitiu entender como está ocorrendo o gerenciamento de projetos na empresa e responder à questão de pesquisa. Adicionalmente, permitiu que fosse delineado o framework híbrido apresentado nesta seção, representando o estágio de aplicação dos processos ágeis e tradicionais no gerenciamento dos projetos de infraestrutura de TI da empresa. 
Os resultados demonstraram que os principais fatores que contribuíram para moldar o framework híbrido atualmente em progresso na empresa foram as barreiras organizacionais e operacionais, que praticamente exigem a permanência dos processos de gerenciamento tradicionais nas fases iniciais e finais dos projetos. Nesse contexto, o gerenciamento ágil não é uma realidade plena nos projetos de infraestrutura de TI, devido a características como a necessidade de processos controlados e documentação obrigatória, evidenciando que a transformação ágil precisa se adequar à realidade das diversas áreas da empresa.

A coincidência nas respostas dos entrevistados mostrou que os processos tradicionais adequam-se e estão sendo aplicados nas fases iniciais e finais dos projetos: análise de viabilidade, iniciação, planejamento e encerramento. Por outro lado, os métodos ágeis revelam-se mais adequados para aplicação nas fases intermediárias, em ciclos de execução ou implantação. A Figura 3 representa esta situação.

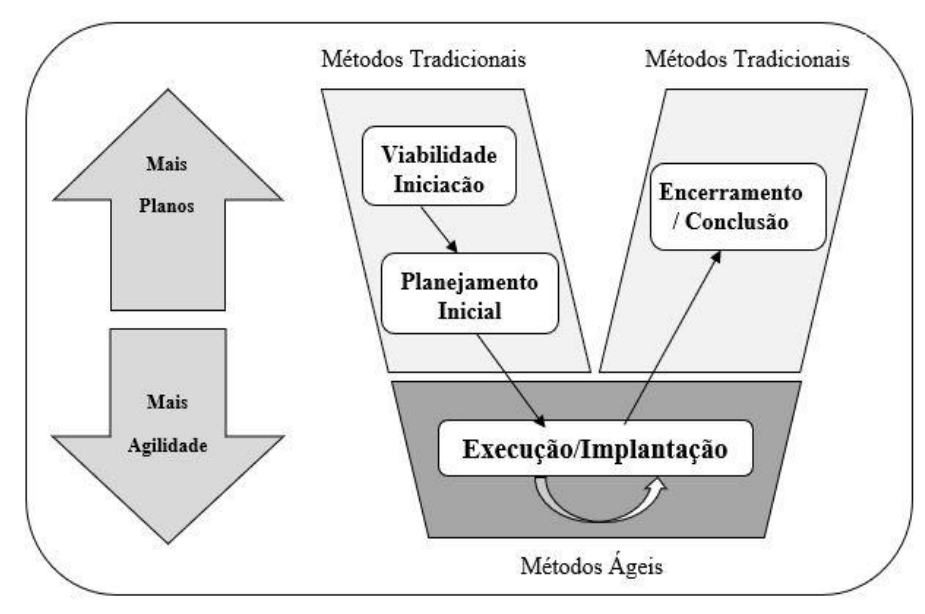

Figura 3 - Framework híbrido proposto para a situação de transição Fonte: Adaptado pelos autores de Hayata e Han (2011).

\section{O framework híbrido aqui proposto} segue, na prática, as características da concepção teórica apresentada por Hayata e Han (2011) em seu Modelo-V, também descrita por Rahmanian (2014). O modelo original desses autores representa uma combinação de práticas da metodologia ágil SCRUM e da metodologia tradicional. Aqui, esse modelo foi adaptado aos processos utilizados na área de infraestrutura de TI empresa, informados pelos entrevistados. No Modelo-V, os níveis "mais altos" de abstração são representados na parte superior do diagrama. Aqui, este nível corresponde às atividades relacionadas com o aspecto de planejamento dos métodos tradicionais, como iniciação, requisitos, custos, alocação de recursos, negociação com gestores e 
encerramento do projeto. Já os níveis "mais baixos" de abstração são representados na parte inferior do diagrama. Este nível corresponde aqui às atividades com as características de flexibilidade e rapidez dos métodos ágeis, como os processos cíclicos de planejamento e execução ou implantação da infraestrutura de TI propriamente dita.

\section{Considerações finais}

Este artigo foi desenvolvido como um estudo de caso único, analisando o processo de transformação ágil de uma empresa de Telecom, que está mudando seu modelo de gestão de projetos. O objetivo principal do artigo foi descrever o processo de transformação ágil e identificar o framework híbrido de gerenciamento de projetos existente na conjuntura da transição. Paralelamente, evidenciou-se como esse contexto foi moldado pelos elementos facilitadores e os obstáculos ao processo de implantação. A unidade de análise foi o departamento de infraestrutura de TI da empresa, que sempre utilizou o gerenciamento tradicional e está se adaptando para adotar o gerenciamento ágil. Os dados foram coletados por meio de entrevistas com quatro gerentes de projeto e um arquiteto de soluções de infraestrutura de TI, análise documental e observação participante por parte de um dos autores, envolvido no processo de implantação. Os entrevistados são profissionais experientes em gerenciamento de projetos tradicional, com experiências distintas em gestão de projetos ágeis. Todos, porém, foram enfáticos em considerar a dinâmica dos métodos ágeis bastante válida, constituindo-se hoje em uma realidade na área de gestão de projetos, tanto na empresa quanto no mercado.

Os resultados mostram que a transformação ágil se encontra em diferentes estágios dentro da área analisada. Para alguns projetos o processo ainda não começou, outros estão em preparação, outros estão em fase de adaptação aos novos métodos, enquanto outros já estão sendo executados dentro da nova metodologia. As respostas dos entrevistados também evidenciaram que os processos dos projetos estão sendo gerenciados de forma híbrida, parte no modelo tradicional e parte no modelo ágil. Os processos da fase de execução e implantação adequam-se às práticas e métricas ágeis. Neles, é possível quebrar as entregas do produto em partes menores, permitindo ações rápidas para acertos e modificações. Nas fases iniciais e de encerramento do projeto, porém, ainda se aplica o modelo tradicional. Nelas exigem-se documentação extensiva, planos padronizados e processos de aprovação para cumprir determinadas etapas.

Conclui-se, portanto, que o processo de transformação ágil da empresa encontra-se em um momento de transição entre os dois modelos, tradicional e ágil, caracterizando-se como uma gestão híbrida de projetos. A análise desse cenário sugere que o framework híbrido de gestão de projetos identificado é aderente ao Modelo- $\mathrm{V}$, descrito na literatura (Hayata \& Han, 2011), que foi adaptado e 
proposto aqui para representar a situação da empresa.

Entre os facilitadores do processo de transformação ágil foram citados o envolvimento da alta gestão, a adesão das equipes à implantação da nova metodologia, a contratação de uma consultoria especializada, a decisão de estender o processo para as demais áreas da empresa e as ferramentas visuais de controle adotadas. Entre as barreiras, foram mencionadas a forte cultura da utilização dos processos tradicionais, a falta de experiência nos métodos ágeis, as características pouco flexíveis dos projetos de infraestrutura de TI e as exigências legais nos projetos da área governamental.

Este trabalho contribui com o conhecimento de pesquisadores e praticantes da área de gerenciamento de projetos sobre a convivência das abordagens gerenciais nos processos de transformação ágil. Adicionalmente, contribui para aumentar o conhecimento sobre projetos de infraestrutura, um assunto pouco abordado na literatura referente a projetos de TI, mais concentrada em projetos relacionados à área de software. Como limitação, ressaltamos o fato de a pesquisa ter se restringido ao contexto de uma só empresa do ramo de Telecom e a uma só unidade de análise, a área de infraestrutura de TI.

O gerenciamento de projetos híbridos é um tema relativamente novo e com potencial para o desenvolvimento de novos estudos. Futuras pesquisas podem analisar outras áreas dentro da mesma empresa ou mesmo em mais de uma empresa que esteja passando pelo mesmo processo. Outros setores de negócio também podem ser investigados. Além disso, pode ser considerado um recorte temporal mais amplo, com a realização de várias rodadas de entrevistas durante um período mais longo de tempo, se possível acompanhando todo o processo.

\section{Referências}

Adelakun, O., Garcia, R., Tabaka, T., \& Ismail, R. (2017). Hybrid project management: Agile with discipline. International Conference on Information Resources Management (CONF-IRM). Association For Information Systems

Aguanno, K. (2005). Managing agile projects. Canadá: Multi-Media Publications Inc, 415.

Amaral, D. C., Conforto, E. C., Benassi, J. L. G., \& Araujo, C. D. (2011). Gerenciamento ágil de projetos: aplicação em produtos inovadores. São Paulo: Saraiva, 240.

Augustine, S. (2005). Managing agile projects. USA: Prentice Hall PTR.

Babenko, V., Lomovskykh, L., Oriekhova, A., Korchynska, L., Krutko, M., \& Koniaieva, Y. (2019). Features of methods and models in risk management of IT projects.

Periodicals of Engineering and Natural Sciences, 7(2), 629-636. http://dx.doi.org/10.21533/pen.v7i2.558.g33 $\underline{0}$

Bardin, L. (1977). Análise de conteúdo. Lisboa: edições, 70, 225.

Beck, K., Cunningham, W., Hunt, A., Martin, R., Thomas, D., Beedle, M., \& Sutherland, J. (2001). Manifesto ágil. Manifesto para Desenvolvimento Ágil de Software. Retrieved from http://manifestoagil. com. $\underline{\mathrm{br} /}$ 
Bianchi, M. J. (2017). Ferramenta para configuração de modelos híbridos de gerenciamento de projetos. Master's Dissertation, Escola de Engenharia de São Carlos, University of São Paulo, São Carlos. doi:10.11606/D.18.2017.tde-25092017142303. Retrieved 2020-12-16, from www.teses.usp.br

https://doi.org/10.11606/D.18.2017.tde$\underline{25092017-142303}$

Boehm, B. (2002). Get ready for agile methods, with care. Computer, 35(1), 6469.

https://doi.org/10.1109/2.976920

Boehm, B., \& Turner, R. (2003). Using risk to balance agile and plan-driven methods. Computer, 36(6), 57-66. https://doi.org/10.1109/MC.2003.1204376

Burman, E. (2015). Agile in action: Hybrid methodologies in practice. Retrieved from

https://www.divaportal.org/smash/get/diva2:849591/FULLT EXT01.pdf

Cegarra-Navarro, J.-G., Soto-Acosta, P., \& Wensley, A. K. (2016). Structured knowledge processes and firm performance: The role of organizational agility. Journal of Business Research, 69(5), 1544-1549. https://doi.org/10.1016/j.jbusres.2015.10.01 $\underline{4}$

Chin, G. (2004). Agile project management: How to succeed in the face of changing project requirements. New York, NY: Amacom.

Conforto, E. C., Salum, F., Amaral, D. C., Da Silva, S. L., \& De Almeida, L. F. M. (2014). Can agile project management be adopted by industries other than software development? Project Management Journal, 45(3), 21-34. https://doi.org/10.1002/pmj

Cram, W. A., \& Marabelli, M. (2018). Have your cake and eat it too? Simultaneously pursuing the knowledge-sharing benefits of agile and traditional development approaches. Information and Management, 55(3), 322-339.
Creswell, J. W., \& Poth, C. N. (2016). Qualitative inquiry and research design: Choosing among five approaches. Sage publications.

Da Silva, A. B., Godoy, C. P. V., Godoi, C. K., Balsini, C., De Freitas, H. M. R., Macke, J., ... Boeira, S. L. (2017). Pesquisa qualitativa em estudos organizacionais. São Paulo: Saraiva.

DeCarlo, D. (2010). Extreme project management: Using leadership, principles, and tools to deliver value in the face of volatility. USA: John Wiley \& Sons.

Dikert, K., Paasivaara, M., \& Lassenius, C. (2016). Challenges and success factors for large-scale agile transformations: A systematic literature review. Journal of Systems and Software, 119, 87-108.

https://doi.org/10.1016/j.jss.2016.06.013

Dyba, T., \& Dingsoyr, T. (2009). What do we know about agile software development? IEEE Software, 26(5), 6-9.

Eder, S., Conforto, E. C., Amaral, D. C., \& Silva, S. L. da. (2015). Diferenciando as abordagens tradicional e ágil de gerenciamento de projetos. Production, 25(3), 482-497.

Fernandez, D. J., \& Fernandez, J. D. (2008). Agile project management-Agilism versus traditional approaches. Journal of Computer Information Systems, 49(2), 10-17.

Flick, U. (2014). An introduction to qualitative research. California: Sage Publications Inc.

Gandomani, T. J., \& Nafchi, M. Z. (2015). An empirically-developed framework for Agile transition and adoption: A Grounded Theory approach. Journal of Systems and Software, 107, 204-219.

https://doi.org/10.1016/j.jss.2015.06.006

Gill, A. Q., Henderson-Sellers, B., \& Niazi, M. (2018). Scaling for agility: A reference model for hybrid traditional-agile software development methodologies. Information Systems Frontiers, 20(2), 315-341. https://doi.org/10.1007/s10796-016-9672-8 
Gurd, B., \& Ifandoudas, P. (2014). Moving towards agility: the contribution of a modified balanced scorecard system. Measuring Business Excellence, 18(2), 113. https://doi.org/10.1108/MBE-10-20120052

Hayata, T., \& Han, J. (2011). A hybrid model for IT project with Scrum. Proceedings of 2011 IEEE International Conference on Service Operations, Logistics and Informatics, 285-290. IEEE.

https://doi.org/10.1109/SOLI.2011.5986572

Howard, W. R. (2010). Agile Project Management: Creating Innovative Products, Kybernetes, 39(1), 155-155. https://doi.org/10.1108/03684921011021336

Hoda, R., \& Noble, J. (2017). Becoming agile: A grounded theory of agile transitions in practice. 2017 IEEE/ACM 39th International Conference on Software Engineering (ICSE), 141-151. IEEE. https://doi.org/10.1109/ICSE.2017.21

Kusters, R. J., van de Leur, Y., Rutten, W. G., \& Trienekens, J. J. (2017, April). When agile meets waterfall-investigating risks and problems on the interface between agile and traditional software development in a hybrid development organization. In International Conference on Enterprise Information Systems (Vol. 2, pp. 271-278). SCITEPRESS.

https://doi.org/10.5220/0006292502710278

Kvale, S. (2008). Doing Interviews. USA: SAGE Publications Inc.

Leffingwell, D. (2011). Agile software requirements: Lean requirements practices for teams, programs, and the enterprise. USA: Addison-Wesley Professional.

Meirinhos, M., \& Osório, A. (2016). O estudo de caso como estratégia de investigação em educação. EduSer-Revista de educação, 2(2). http://dx.doi.org/10.34620/ed

Moe, N. B., \& Dingsøyr, T. (2017). Emerging research themes and updated research agenda for large-scale agile development: A summary of the 5th international workshop at XP2017. Proceedings of the XP2017

Scientific Workshops, 1-4. https://doi.org/10.1145/3120459.3120474

Nerur, S., Mahapatra, R., \& Mangalaraj, G. (2005). Challenges of migrating to agile methodologies. Communications of the ACM, 48(5), 72-78.

Paterek, P. (2018). Agile Transformation Framework in Software Project Organization. ICMLG 2018: 6th International Conference on Management, Leadership and Governance, 258-267.

Patton, M. Q. (2005). Qualitative research. Encyclopedia of statistics in behavioral science.

https://doi.org/10.1002/0470013192.bsa514

Ponelis, S. R. (2015). Using interpretive qualitative case studies for exploratory research in doctoral studies: A case of information systems research in small and medium enterprises. International Journal of Doctoral Studies, 10, 535-550.

Pressman, R., \& Maxim, B. (2016). Engenharia de Software-8a Edição. McGraw Hill Brasil.

Qu, S. Q., \& Dumay, J. (2011). The qualitative research interview. Qualitative Research in Accounting and Management, 8(3), 238264.

https://doi.org/10.1108/11766091111162070

Rahmanian, M. (2014). A Comparative Study on Hybrid IT Project Managment Using Traditional Project Management and Agile Approach. International Journal of Computer and Information Technology, 03(05), 1096-1099.

Ravichandran, T. (2018). Exploring the relationships between IT competence, innovation capacity and organizational agility. The Journal of Strategic Information Systems, 27(1), 22-42. 
Shenhar, A. (2015). What is strategic project leadership?. Open Economics and Management Journal, 2(1). https://doi.org/10.2174/23526300015020100 $\underline{29}$

Shenhar, A. J., \& Dvir, D. (2010).

Reinventando gerenciamento de projetos: a abordagem diamante ao crescimento e inovação bem-sucedidos. São Paulo: M. Books.

Silva, F. B., Bianchi, M. J., \& Amaral, D. C. (2019). Evaluating combined project management models: Strategies for agile and plan-driven integration. Product: Management and Development, 17(1), 1530. http://dx.doi.org/10.4322/pmd.2019.003
Sweetman, R., \& Conboy, K. (2018). Portfolios of Agile Projects: A Complex Adaptive Systems' Agent Perspective. Project Management Journal, 49(6), 18-38. https://doi.org/10.1177/8756972818802712

Vinekar, V., Slinkman, C. W., \& Nerur, S. (2006). Can agile and traditional systems development approaches coexist? An ambidextrous view. Information Systems Management, 23(3), 31-42.

Wysocki, R. K. (2019). Effective project management: Traditional, agile, extreme. John Wiley \& Sons.

Yin, R. K. (2015). Estudo de Caso: Planejamento e métodos. São Paulo: Bookman. 